\title{
ON THE OPTIMUM GRADIENT METHOD FOR SYSTEMS OF LINEAR EQUATIONS
}

\author{
M. MARCUS
}

In the optimum gradient procedure for solving the system of linear equations

$$
A x=b
$$

the choice of metric is arbitrary. In this note we consider a comparison of metrics induced by certain matrix functions of $A$ in terms of rapidity of convergence. $A$ is an $n$-square, nonsingular, complex matrix, $x$ and $b$ are complex, column $n$-vectors, $z^{*}$ is the conjugate transpose of $z, \lambda(A)$ denotes an arbitrary eigenvalue of $A$, and $\lambda_{m}(A)$ and $\lambda_{M}(A)$ are the minimum and maximum eigenvalues of $A$ respectively when all $\lambda(A)$ are real. In this case we denote by $I_{A}$ the closed interval $\left[\lambda_{m}(A), \lambda_{M}(A)\right]$.

If $R$ is a positive-definite Hermitian (p.d.h.) matrix define

$$
\|z\|_{R}^{2}=z^{*} R z .
$$

Solving (1) is then equivalent to minimizing the function

$$
\phi(x)=\|A x-b\|_{R}^{2} .
$$

Denote by $L(x, d)$ the set of vectors $x-\alpha d$ for $\alpha \in(-\infty, \infty)$. In [2] the following relations are established:

$$
\min _{x \in L\left(x_{0}, d\right)} \phi(x)=\phi\left(x_{0}-\alpha_{0} d\right)
$$

where $\alpha_{0}=\operatorname{Re}\left(d^{*}\left(B x_{0}-c\right) / d^{*} B d\right), B=A^{*} R A, c=A^{*} R b$; and

$$
\phi\left(x_{0}\right)-\phi\left(x_{0}-\alpha_{0} d\right)=\alpha_{0}^{2} d^{*} B d .
$$

$\alpha_{0}$ is called the optimum $\alpha$ in direction $d$ at $x_{0}$. Note that $B$ is p.d.h. In [2] the optimum gradient procedure for solving (1) is defined as follows:

(i) let $x_{0}$ be arbitrary,

(ii) set $x_{k+1}=x_{k}-\alpha_{k} z_{k}$ where $z_{k}=B x_{k}-c$ and $\alpha_{k}$ is the optimum $\alpha$ in direction $z_{k}$ at $x_{k}$. From (5) we see that

Received by the editors January 28, 1955. 


$$
\phi\left(x_{k+1}\right)=\phi\left(x_{k}\right)-\alpha_{k}^{2} z_{k}^{*} B z_{k}
$$

and hence

$$
\begin{aligned}
\frac{\phi\left(x_{k+1}\right)}{\phi\left(x_{k}\right)} & =1-\alpha_{k} \frac{z_{k}^{*} z_{k}}{\phi\left(x_{k}\right)} \\
& =1-\frac{z_{k}^{*} z_{k}}{z_{k}^{*} B z_{k}} \frac{z_{k}^{*} z_{k}}{z_{k}^{*} B^{-1} z_{k}} \\
& \leqq 1-\frac{4}{\left(\left(\lambda_{M}(B) / \lambda_{m}(B)\right)^{1 / 2}+\left(\lambda_{m}(B) / \lambda_{M}(B)\right)^{1 / 2}\right)^{2}}
\end{aligned}
$$

This last inequality is due to L. V. Kantorovich [3], and is proved by maximizing the function

$$
\left(\sum_{k=1}^{n} \lambda_{k} u_{k}^{2}\right)\left(\sum_{k=1}^{n} \lambda_{k}^{-1} u_{k}^{2}\right),
$$

subject to the restraint

$$
\sum_{k=1}^{n} u_{k}^{2}=1
$$

The results here will be stated for $A$ p.d.h. Let $\psi(x)$ be a differentiable function for $x \in I_{A}$ and we may define $\psi(A)$ as in [1]. For, since $A$ is p.d.h. there exists $U$, unitary, such that $U^{-1} A U$ is the direct sum of scalar matrices $\lambda_{1} I_{1}, \cdots, \lambda_{e} I_{0}$. We then define $U^{-1} \psi(A) U$ as the direct sum of the matrices $\psi\left(\lambda_{1}\right) I_{1}, \cdots, \psi\left(\lambda_{e}\right) I_{e}$. We shall assume $\psi(x)$ is real, $\psi^{*}(A)=\psi\left(A^{*}\right)$, and $\psi(A)$ is nonsingular, $\psi(\lambda(A)) \neq 0$.

Set

$$
R_{\psi}=\psi^{*}(A) \psi(A)=\psi\left(A^{*}\right) \psi(A)
$$

and

$$
B_{\psi}=A^{*} R_{\psi} A=A^{*} \psi\left(A^{*}\right) A \psi(A) .
$$

$R_{\psi}$ defines a metric as in (2) since $\psi(\lambda(A)) \neq 0$. Define

$$
\begin{aligned}
\mu(B) & =\lambda_{m}(B) / \lambda_{M}(B), \\
\sigma(B) & =\frac{4}{\left(\left(\lambda_{M}(B) / \lambda_{m}(B)\right)^{1 / 2}+\left(\lambda_{m}(B) / \lambda_{M}(B)\right)^{1 / 2}\right)^{2}},
\end{aligned}
$$

and

$$
\alpha_{\psi}=\frac{\sigma\left(B_{\psi}\right)}{\sigma\left(B_{1}\right)}
$$


In view of (6) we shall say that $R_{\psi}$ is as fast a metric as $I$ when $\alpha_{\psi} \geqq 1$.

THEOREM 1. If

$$
-\frac{\psi^{2}(x)}{x} \leqq \psi(x) \psi^{\prime}(x) \leqq 0
$$

for $x \in I_{A}$, then $R_{\psi}$ is as fast a metric as $I$.

Proof. From (7) and (8) we have

$$
\sigma\left(B_{\psi}\right)=\frac{4 \mu\left(B_{\psi}\right)}{\left(1+\mu\left(B_{\psi}\right)\right)^{2}} .
$$

Set $f_{\psi}(x)=x^{2} \psi^{2}(x)$ and then

$$
B_{\psi}=A^{2} \psi^{2}(A)=f_{\psi}(A) .
$$

A p.d.h. and (10) together imply

$$
f_{\psi}^{\prime}(x)=2 x\left(\psi^{2}(x)+x \psi(x) \psi^{\prime}(x)\right) \geqq 0
$$

and thus $f_{\psi}(x)$ is monotonic increasing in $I_{A}$. Similarly $\psi^{2}(x)$ is monotonic decreasing in $I_{A}$. To simplify the notation set

$$
\sigma\left(B_{\psi}\right)=\sigma_{\psi}, \quad \omega_{\psi}=\frac{\psi^{2}\left(\lambda_{m}(A)\right)}{\psi^{2}\left(\lambda_{M}(A)\right)}, \quad \bar{\mu}=\mu^{2}(A)
$$

and then

$$
\begin{aligned}
\frac{\sigma_{\psi}}{4} & =\frac{\lambda_{m}\left(f_{\psi}(A)\right) / \lambda_{M}\left(f_{\psi}(A)\right)}{\left(1+\lambda_{m}\left(f_{\psi}(A)\right) / \lambda_{M}\left(f_{\psi}(A)\right)\right)^{2}} \\
& =\frac{\min _{\lambda(\Lambda)} f_{\psi}(\lambda(A)) / \max _{\lambda(\Lambda)} f_{\psi}(\lambda(A))}{\left(1+\min _{\lambda(\Lambda)} f_{\psi}(\lambda(A)) / \max _{\lambda(\boldsymbol{A})} f_{\psi}(\lambda(A))\right)^{2}} \\
& =\frac{\bar{\mu} \omega_{\psi}}{\left(1+\bar{\mu} \omega_{\psi}\right)^{2}} \cdot
\end{aligned}
$$

The condition

$$
\alpha_{\psi} \geqq 1
$$

is then equivalent to

$$
\frac{\bar{\mu} \omega_{\psi}}{\left(1+\bar{\mu} \omega_{\psi}\right)^{2}} \geqq \frac{\bar{\mu}}{(1+\bar{\mu})^{2}}
$$




$$
\vec{\mu}^{2} \omega_{\psi}^{2}-\omega_{\psi}\left(\bar{\mu}^{2}+1\right)+1 \leqq 0 .
$$

Noting that $\mu \leqq 1$, (11) becomes

$$
1 \leqq \omega_{\psi} \leqq 1 / \bar{\mu}^{2}
$$

which in turn reduces to

$$
\mu^{4}(A) \psi^{2}\left(\lambda_{M}(A)\right) \leqq \psi^{2}\left(\lambda_{m}(A)\right) \mu^{4}(A) \leqq \psi^{2}\left(\lambda_{M}(A)\right) .
$$

Substituting in the last inequality of (12) yields

$$
\begin{aligned}
\psi^{2}\left(\mu(A) \lambda_{M}(A)\right) \mu^{4}(A) & \leqq \psi^{2}\left(\lambda_{M}(A)\right), \\
\frac{\mu^{2}(A)}{\lambda_{M}(A)}\left(\mu(A) \lambda_{M}(A)\right)^{2} \psi^{2}\left(\mu(A) \lambda_{M}(A)\right) & \leqq \psi^{2}\left(\lambda_{M}(A)\right),
\end{aligned}
$$

and finally

$$
\mu^{2}(A) f_{\psi}\left(\mu(A) \lambda_{M}(A)\right) \leqq f_{\psi}\left(\lambda_{M}(A)\right) .
$$

The monotonicity of $f_{\psi}$ implies (13) and the left side of (12) follows similarly.

For example, if

$$
\psi(x)=x^{\alpha}, \quad-1 \leqq \alpha \leqq 0,
$$

(10) clearly holds.

It is of interest to obtain an estimate of $\alpha_{\psi}$ solely in terms of $\psi$ and the entries of $A$.

Theorem 2. Assume the conditions of Theorem 1. Let $i_{1} \leqq i_{2} \leqq \ldots$ $\leqq i_{k}, k \leqq n$, be a sequence of positive integers and set

$$
\begin{aligned}
S\left(i_{1}\right. & \left.\cdots, i_{k}\right)=\frac{1}{k} \sum_{\alpha, v=1}^{k} A_{i_{\alpha^{i}},}, \\
M & =\max S\left(i_{1}, \cdots, i_{k}\right), \\
m & =\min S\left(i_{1}, \cdots, i_{k}\right), \\
\mu_{0} & =(m / M)^{2},
\end{aligned}
$$

and

$$
\delta=\psi^{2}(m) / \psi^{2}(M)
$$

Then

$$
\alpha_{\psi} \geqq \frac{\delta}{\left(1+\mu_{0} \delta\right)^{2}}
$$

Proof. Note that 


$$
\begin{aligned}
& \lambda_{M}(A)=\max _{z^{*} z=1} z^{*} A z, \\
& \lambda_{m}(A)=\min _{z^{*} z=1} z^{*} A z,
\end{aligned}
$$

and hence $z^{*} A z \in I_{A}$ for $z^{*} z=1$. Let $z_{0}$ be the unit vector with $1 / k^{1 / 2}$ in the $i_{j}$ position, and 0 elsewhere. It is easy to check that

$$
z_{0}^{*} A z_{0}=S\left(i_{1}, \cdots, i_{k}\right) \in I_{A} .
$$

It follows from the monotonicity properties of $\psi$ and $f_{\psi}$ that

$$
\begin{aligned}
\alpha_{\psi} & =\frac{\omega_{\psi}}{\left(1 /(1+\bar{\mu})+(\bar{\mu} /(1+\bar{\mu})) \omega_{\psi}\right)^{2}} \\
& \geqq \frac{\omega_{\psi}}{\left(1+\bar{\mu} \omega_{\psi}\right)^{2}} \\
& \geqq \frac{\psi^{2}(m) / \psi^{2}(M)}{\left(1+f_{\psi}(m) / f_{\psi}(M)\right)^{2}} \\
& =\frac{\delta}{\left(1+\mu_{0} \delta\right)^{2}} .
\end{aligned}
$$

We may remark that for

$$
\frac{\delta}{\left(1+\mu_{0} \delta\right)^{2}} \geqq 1
$$

it is necessary that $\mu_{0} \leqq 1 / 4$.

\section{REFERENCES}

1. W. L. Ferrar, Finite matrices, Oxford, 1951, p. 99.

2. A. S. Householder, Principles of numerical analysis, McGraw-Hill, 1953.

3. L. V. Kantorovich, Functional analysis and applied mathematics, Uspehi Matematiðeskih Nauk vol. 3 no. 6 (1948) pp. 89-185.

The University of British Columbia 